\title{
The Effect of Geometric Configurations on the Elastic Behavior of an Edge-Cracked Bonded Strip
}

\author{
Xin Lan, ${ }^{1}$ Shaobo Ji, ${ }^{1}$ Nao-Aki Noda, ${ }^{2}$ and Yong Cheng ${ }^{1}$ \\ ${ }^{1}$ School of Energy and Power Engineering, Shandong University, 17923 Jingshi Road, Jinan, Shandong, China \\ ${ }^{2}$ Department of Mechanical Engineering, Kyushu Institute of Technology, 1-1 Sensui-cho, Tobata-ku, Kitakyushu-shi, \\ Fukuoka 804-8550, Japan \\ Correspondence should be addressed to Xin Lan; xinlan_al@yahoo.com
}

Received 2 January 2017; Accepted 9 February 2017; Published 26 March 2017

Academic Editor: Giorgio Pia

Copyright (C) 2017 Xin Lan et al. This is an open access article distributed under the Creative Commons Attribution License, which permits unrestricted use, distribution, and reproduction in any medium, provided the original work is properly cited.

\begin{abstract}
Interface cracks often initiate around the bonding free-edge corner due to the high-stress concentration. In this research, the elastic behavior of an edge-cracked dissimilar bonded strip subjected to remote tensile load is investigated using the proportional crack tip opening displacement method based on FE analysis for arbitrary material combinations. The stress intensity factor, energy release rate, and mode mixity are computed and compared systematically with varying geometrical configurations and material combinations. Then, the combined effects of the relative height of the bonded component and material combination are discussed for the typical engineering materials.
\end{abstract}

\section{Introduction}

There is an increasing demand on the use of bonded joints in modern structural applications. Interfacial cracks are normally observed around the bonding edge region in joints and areas of discontinuities due to the high-stress concentration, residual stresses, and interfacial flaws. The presence of a crack affects the performance of a structure, and the propagation may eventually cause a through thickness crack which results in the failure of a structure.

The problem of interface crack was firstly investigated by Williams [1] who discovered the asymptotic solution of the elastic field around an open crack; then, his work was followed and extended by Rice and Sih [2], Erdogan [3, 4], England [5], Willis [6], and many others. In linear elastic fracture mechanics, the stress intensity factor (SIF) and energy release rate (ERR) are used to predict the stress state and the stable crack growth caused by the remote load. Therefore, many researchers have tried to solve the singular fields for various geometries and material combinations. Recently, many studies were found to accurately determine the order of the stress singularity [7-12]. Then, quite a lot of researches have been devoted to the analysis of the intensity of the singular stress fields for interfacial crack problems $[13,14]$. Akisanya and Fleck [15] evaluated the singular stress fields at the free edge of a long bimaterial strip subjected to uniform tension. Lan et al. [16] investigated the effect of material combinations and the relative crack size on the SIFs at the crack tip of a bimaterial bonded strip. Noda and Lan [17] analyzed the SIFs of the shallow edge interface cracks for arbitrary material combinations and proposed an empirical formula for the equal pair zone in the $\alpha-\beta$ space.

However, all the aforementioned studies concentrate their discussions on the case of bonded long strips and ignore the effect of geometrical configurations. In the practical industrial applications, the geometrical configuration of the bonded strip varies greatly with the actual demands. The different heights of the two bonded components may exhibit longitudinal symmetry or asymmetry characteristics, thus producing the variation of the mixed mode for a specific material combination. As known, the fracture toughness (i.e., critical energy release rate or critical stress intensity factor) depends strongly on the mode mixity. In order to predict the onset of the delamination at the bimaterial interface, one must investigate the elastic behaviors for various geometric configurations. In this research, the SIFs, ERR, and 
mix-mode angle are systematically computed for various geometric configurations and material combinations based on $\mathrm{FE}$ analysis using the proportional crack tip opening displacement (CTOD) method [18]. The relative height of the two components of the bonding strip varies from $l / W=2$ to $l / W=0.333$, and the geometric configurations are composed of longitudinal symmetrical and asymmetrical cases. Then, the effects of the heights of the bonded components together with material combinations are thoroughly investigated for the most fundamental edge interface crack problems. The conclusion may help to understand the dependence of the fracture mechanism on the geometric configurations and may also contribute to the guidance for material selection and structural design for the modern layered materials.

\section{Analysis Method}

2.1. Formulation for the Interface Crack Problems. Consider two isotropic elastic materials joined along the $x$-axis as indicated in Figure 1 with material 1 above the interface and material 2 below. The stress distributions along the interface are defined as shown in [19]

$$
\sigma_{y}+i \tau_{x y}=\frac{K_{\mathrm{I}}+i K_{\mathrm{II}}}{\sqrt{2 \pi r}}\left(\frac{r}{2 a}\right)^{i \varepsilon}, \quad r \longrightarrow 0 .
$$

Here, $\sigma_{y}$ and $\tau_{x y}$ denote the normal and shear stress components near the crack tip, respectively, $r$ is the radial distance behind the crack tip, $a$ is the half crack length, and $\varepsilon$ is the bielastic constant given by

$$
\begin{aligned}
\varepsilon & =\frac{1}{2 \pi} \ln \left[\frac{\left(\kappa_{1} / \mu_{1}+1 / \mu_{2}\right)}{\left(\kappa_{2} / \mu_{2}+1 / \mu_{1}\right)}\right], \\
\kappa_{m} & = \begin{cases}3-4 v_{m} & \text { (plane strain) } \\
\frac{3-v_{m}}{1+v_{m}} & \text { (plane stress) }\end{cases}
\end{aligned}
$$

where $\mu_{m}(m=1,2)$ and $\nu_{m}(m=1,2)$ are the shear moduli and Poisson's ratios of either respective material. The associated relative crack opening displacement values $\delta_{d}=$ $u_{d}(r, \theta=\pi)-u_{d}(r, \theta=-\pi)$ and $(d=x, y)$ for nodes $i, i^{\prime}$ at a distance $r$ behind the crack tip, shown in Figure 1, are given by [20]

$$
\begin{aligned}
\delta_{y}+i \delta_{x}= & \frac{K_{\mathrm{I}}+i K_{\mathrm{II}}}{2(1+2 i \varepsilon) \cosh (\varepsilon \pi)}\left[\frac{\kappa_{1}+1}{\mu_{1}}+\frac{\kappa_{2}+1}{\mu_{2}}\right] \\
& \cdot\left(\frac{r}{2 \pi}\right)^{1 / 2}\left(\frac{r}{l}\right)^{i \varepsilon},
\end{aligned}
$$

where $l$ is an arbitrary reference length which scales with specimen size or crack length; for the definition of (1), we have $l=2 a$ without loss of generality.

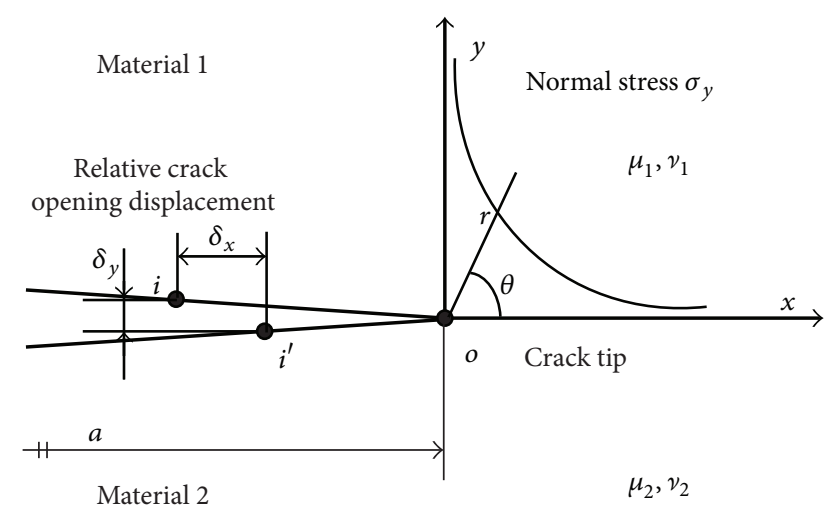

FIGURE 1: Stress distribution and relative crack displacement of an interface crack.

Considering $(r / l)^{i \varepsilon}=\cos (\varepsilon \ln (r / l))+i \sin (\varepsilon \ln (r / l))$ and rearranging (3), then the stress intensity factor components $K_{\mathrm{I}}, K_{\mathrm{II}}$ can be separated as

$$
\begin{aligned}
K_{\mathrm{I}} & =S\left\{\left(\delta_{y}-2 \varepsilon \delta_{x}\right) \cos \left[\varepsilon \ln \left(\frac{r}{l}\right)\right]\right. \\
& \left.+\left(\delta_{x}+2 \varepsilon \delta_{y}\right) \sin \left[\varepsilon \ln \left(\frac{r}{l}\right)\right]\right\}, \\
K_{\mathrm{II}} & =S\left\{\left(\delta_{x}+2 \varepsilon \delta_{y}\right) \cos \left[\varepsilon \ln \left(\frac{r}{l}\right)\right]\right. \\
& \left.-\left(\delta_{y}-2 \varepsilon \delta_{x}\right) \sin \left[\varepsilon \ln \left(\frac{r}{l}\right)\right]\right\}, \\
S & =\frac{2 \cosh (\varepsilon \pi)(r / 2 \pi)^{-1 / 2}}{\left(\left(\kappa_{1}+1\right) / \mu_{1}+\left(\kappa_{2}+1\right) / \mu_{2}\right)} .
\end{aligned}
$$

We can rewrite (4) and (5) as

$$
\begin{aligned}
\frac{K_{\mathrm{I}}}{\delta_{y}} & =S\left\{(\cos Q+2 \varepsilon \sin Q)+(\sin Q-2 \varepsilon \cos Q) \frac{\delta_{x}}{\delta_{y}}\right\}, \\
\frac{K_{\mathrm{II}}}{\delta_{x}} & \\
& =S\left\{(\cos Q+2 \varepsilon \sin Q)-(\sin Q-2 \varepsilon \cos Q) \frac{\delta_{y}}{\delta_{x}}\right\}, \\
Q & =\varepsilon \ln \left(\frac{r}{l}\right) .
\end{aligned}
$$

From (7) and (8), when $Q, \varepsilon$, and $\delta_{y} / \delta_{x}$ are kept the same for two different interface cracks, then we get a relationship as

$$
\begin{aligned}
\frac{K_{\mathrm{I}}}{\delta_{y}} & =\text { const } \\
\frac{K_{\mathrm{II}}}{\delta_{x}} & =\text { const. }
\end{aligned}
$$

Considering two interface crack Problems $A$ and $B$ (say problems in Figure 2), by satisfying the preconditions as 


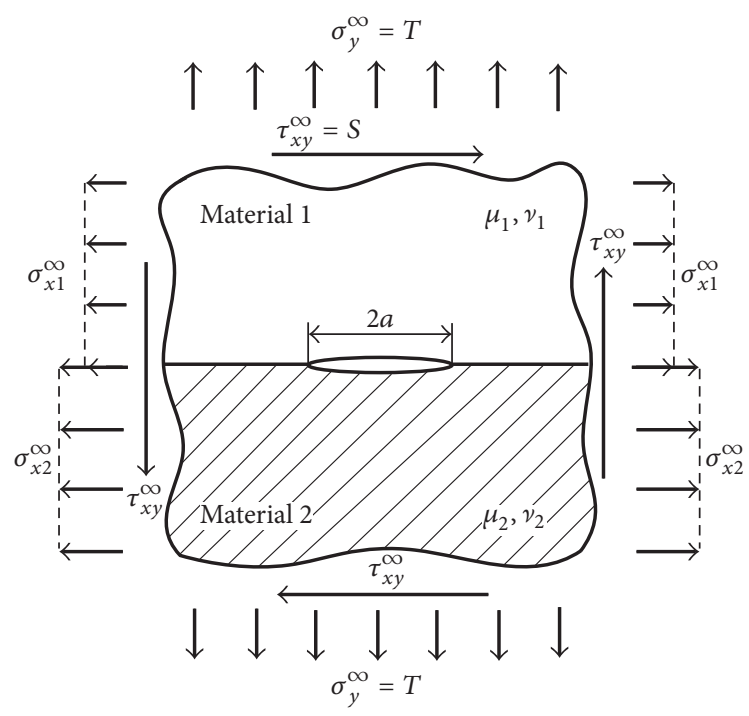

(a)

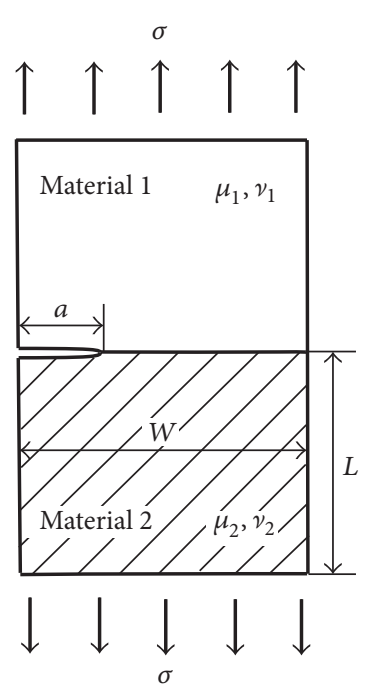

(b)

Figure 2: Geometric configuration for (a) the reference Problem $A$ and (b) the given unknown Problem $B$.

shown in (11) and (12), then the stress intensity factors $K_{\mathrm{I}}, K_{\mathrm{II}}$ exhibit a proportional relationship with $\delta_{y}, \delta_{x}$ as depicted in (13). The relative crack opening displacement values $\delta_{y}$ and $\delta_{x}$ can be computed by FE analysis, assuming one of the two problems is analytically well solved in advance; $K_{\mathrm{I}}$ and $K_{\mathrm{II}}$ of Problem $A$ are given in advance, and then the SIFs of Problem $B$ can be easily obtained from (13). Hence,

$$
\begin{aligned}
&\left(\begin{array}{c}
Q_{A}=Q_{B} \\
\varepsilon_{A}=\varepsilon_{B}
\end{array}\right) \longrightarrow\left(\left[\varepsilon \ln \left(\frac{r}{l}\right)\right]_{A}=\left[\varepsilon \ln \left(\frac{r}{l}\right)\right]_{B}\right), \\
& {\left[\frac{\delta_{y}}{\delta_{x}}\right]_{A}=\varepsilon_{B} } \\
& {\left[\frac{\delta_{y}}{\delta_{x}}\right]_{B}, } \\
& {\left[\frac{K_{\mathrm{I}}}{\delta_{y}}\right]_{A}=\left[\frac{K_{\mathrm{I}}}{\delta_{y}}\right]_{B}, } \\
& {\left[\frac{K_{\mathrm{II}}}{\delta_{x}}\right]_{A}=\left[\frac{K_{\mathrm{II}}}{\delta_{x}}\right]_{B}, }
\end{aligned}
$$

and the strain energy release rate and mode angle for the crack advance in the interface are

$$
\begin{aligned}
& G=\frac{1}{16 \cosh ^{2}(\varepsilon \pi)}\left[\frac{\kappa_{1}+1}{\mu_{1}}+\frac{\kappa_{2}+1}{\mu_{2}}\right]\left(K_{\mathrm{I}}^{2}+K_{\mathrm{II}}^{2}\right), \\
& \psi=\arctan \left[\frac{K_{\mathrm{II}}}{K_{\mathrm{I}}}\right] .
\end{aligned}
$$

2.2. Application of the Proportional COD Method. The problem where SIFs have been solved in advance can be treated as the reference. Therefore, a central cracked dissimilar bonded half plane subjected to remotely uniform tensile and shear stresses as shown in Figure 2(a) is selected as the reference problem for generality. Its analytical solution was firstly derived by Rice and Sih [2] and takes the form

$$
K_{\mathrm{I}}^{*}+i K_{\mathrm{II}}^{*}=\left(\sigma_{y}^{\infty}+i \tau_{x y}^{\infty}\right) \sqrt{\pi a}(1+2 i \varepsilon)
$$

where an asterisk $(*)$ is employed to denote the SIFs for the reference problem. $\sigma_{y}^{\infty}$ and $\tau_{x y}^{\infty}$ are the remote uniform tension and shear applied to the bonded half planes. $a$ is the half crack length of the center crack. Furthermore, the transversal tension values $\sigma_{x 1}^{\infty}$ and $\sigma_{x 2}^{\infty}$ in Figure 2(a) behave as

$$
\begin{aligned}
\sigma_{x 2}^{\infty} & =\frac{1}{1+\kappa_{2}}\left[\frac{\mu_{2}}{\mu_{1}}\left(1+\kappa_{1}\right) \sigma_{x 1}^{\infty}\right. \\
& \left.+\left\{3-\kappa_{2}-\frac{\mu_{2}}{\mu_{1}}\left(3-\kappa_{1}\right) \sigma_{y}^{\infty}\right\}\right] .
\end{aligned}
$$

As previously mentioned, the preconditions in (11) and (12) should be firstly met to ensure the current method is available. Equation (11) can be easily satisfied by making the bielastic constant $\varepsilon$ and the relative distance behind the crack tip $r / l$ the same for the two problems. Here, some extra techniques should be employed to make (12) satisfied. We consider the reference problem shown in Figure 2(a); the relative CODs $\delta_{y}$ and $\delta_{x}$ can be solved in an indirect manner using the principle of linear superposition. Let $\delta_{y, A}^{*}$ and $\delta_{x, A}^{*}$ denote the CODs of Problem $A$ subjected to combined $T$ and $S ; \delta_{y, A 1}^{T=1^{*}}$ and $\delta_{x, A 1}^{T=1^{*}}$ denote those of Problem $A$ subjected to pure unit tension $T=1$; and $\delta_{y, A 2}^{S=1^{*}}$ and $\delta_{x, A 2}^{S=1^{*}}$ denote those of Problem $A$ subjected to pure unit shear $S=1$, respectively. Using the theory of linear superposition, then the relative 
CODs $\delta_{y, A}^{*}$ and $\delta_{x, A}^{*}$ of the reference problem (Problem $A$ ) take the following form :

$$
\begin{aligned}
& \delta_{y, A}^{*}=\delta_{y, A 1}^{T=1^{*}} \times T+\delta_{y, A 2}^{S=1^{*}} \times S, \\
& \delta_{x, A}^{*}=\delta_{x, A 1}^{T=1^{*}} \times T+\delta_{x, A 2}^{S=1^{*}} \times S .
\end{aligned}
$$

Recall (12) and substitute $\delta_{y}$ and $\delta_{x}$ with $\delta_{y, A}^{*}$ and $\delta_{x, A}^{*}$ for Problem $A$; then, we have

$$
\left[\frac{\delta_{y, A}^{*}}{\delta_{x, A}^{*}}\right]_{A}=\left[\frac{\delta_{y, A 1}^{T=1^{*}} \times T+\delta_{y, A 2}^{S=1^{*}} \times S}{\delta_{x, A 1}^{T=1^{*}} \times T+\delta_{x, A 2}^{S=1^{*}} \times S}\right]_{A}=\left[\frac{\delta_{y, B}}{\delta_{x, B}}\right]_{B} .
$$

Rearranging (18) gives the solution of $S / T$ :

$$
\frac{S}{T}=\frac{\delta_{x, B} \cdot \delta_{y, A 1}^{T=1^{*}}-\delta_{y, B} \cdot \delta_{x, A 1}^{T=1^{*}}}{\delta_{y, B} \cdot \delta_{x, A 2}^{S=1^{*}}-\delta_{x, B} \cdot \delta_{y, A 2}^{S=1^{*}}}
$$

Using $T$ and $S$ in (19) as the boundary condition for Problem $A$, then (12) is satisfied and eventually (13) is set up. Finally, the SIFs for the target unknown problem (Problem $B$ ) can be obtained using the proportional relationship as given in (20):

$$
\begin{gathered}
K_{\mathrm{I}, B}=\frac{\delta_{y, B}}{\delta_{y, A}} \times K_{\mathrm{I}, A}, \\
K_{\mathrm{II}, B}=\frac{\delta_{x, B}}{\delta_{x, A}} \times K_{\mathrm{II}, A} .
\end{gathered}
$$

\section{Method Robustness Study}

The computational accuracy of the current procedure is demonstrated by pursuing a method robustness study. The test example is an edge interface crack in plane stress condition. The crack length is set to $2 a=2 \mathrm{~mm}$. A plate width of $W=1620 \times 2 a=3240 \mathrm{~mm}$ and a length of $L=2 W=6480 \mathrm{~mm}$ are used to model the reference problem. The crack length for the given unknown Problem $B$ is fixed to $a=1 \mathrm{~mm}$, and the width of the bonded strip varies in the range $a / W=0.1 \sim 0.4$. The length is set to 2 times the width of the bonded strip. Furthermore, the minimum finite element sizes $e_{\min }=3^{6}$ are kept the same for the reference and the given unknown problems. The same elastic parameters $E_{1} / E_{2}=2,4,10,100$ and $v_{1}=v_{2}=$ 0.3 which were adopted by other researchers [21] are also assumed in the computation. Figure 3 shows finite element idealization with linear quadrilateral elements. The singular regions around the crack tip are well refined in a self-similar manner by increasing the number of layers, and the element size for each inferior layer is one-third the superior one. All the CODs are computed using the MSC.MARC [22] finite element analysis package in this research. The normalized SIFs $K_{\mathrm{I}} / \sigma \sqrt{\pi a}$ and $K_{\mathrm{II}} / \sigma \sqrt{\pi a}$ are tabulated in Table 1 together with those predicted by Matsumto et al. [21]. As shown in this table, the results of the current procedure coincide with those predicted by Matsumto et al. [21].

\section{Results and Discussions}

4.1. Geometric Configurations and Material Elastic Parameters. The geometric configurations of the edge-cracked bimaterial strips are shown in Figure 4. Two elastic, isotropic, and homogenous strips are perfectly bonded along the interface with material 1 above and material 2 below. Let $a$ denote the interface crack length, $W$ denote the width of the bonded strip, and $L_{1}$ and $L_{2}$ denote the height of each material, respectively. There are two geometric configurations considered in this research. Namely, they are the symmetrically and asymmetrically bonded strip shown in Figures 4(a) and 4(b), respectively. The heights of the two bonded components 1 and 2 in Figure 4(a) are kept the same and are variable $L_{1}=L_{2}=0.333 W \sim 2 W$. However, in Figure 4(b), only the height of the upper component 1 varies in the range $L_{1}=0.333 W \sim 2 W$, leaving the lower component 2 fixed to $L_{2}=2 W$. Furthermore, in this research, the relative crack length is fixed to a constant of $a / W=0.1$ to make the effect of geometric configurations obvious. Then, the SIFs, ERRs, and mode angles are computed for the two different geometric configurations with varying height of the bonded materials.

The singular stress fields around the crack tip for the aforementioned problems with a given geometric configuration are only determined on the two elastic mismatch parameters $(\alpha, \beta)$ [24]. The physically admissible values are allowed to lie within a parallelogram dominated by $\alpha= \pm 1$ and $\beta=\alpha / 4 \pm 0.25$. The representative material combinations for the typical engineering materials were firstly compiled by Suga et al. [23]. They pointed out that the $(\alpha, \beta)$ values for typical material combinations are concentrated along $\beta=0$ and $\beta=\alpha / 4$ lines in $\alpha-\beta$ space. Here, we plot the material combinations using the data compiled by Suga et al. [23] together with those provided by others in Figure 5. It can be noted that most of the combinations fall within a narrow band in $\beta=\alpha / 2 \pm 0.1$, and the $(\alpha, \beta)$ values of typical material combinations are distributed in the vicinity of $\beta=0.325 \alpha$ and $\beta=0.25 \alpha$ for plane stress and plane strain conditions, respectively. Therefore, in this research, the SIFs, ERRs, and mode angles are systematically computed for the whole physical admissible $(\alpha, \beta)$ as well as the typical engineering material combinations $\beta=0.325 \alpha$ and $\beta=$ $0.25 \alpha$.

4.2. Solutions of the Bimaterial Bonded Long Strip. As a reference, the bimaterial bonded long strip subjected to tension shown in Figure 4(a) is firstly investigated. The heights of the two components are long enough $(L / W \geq 2)$ to make sure that the crack tip fields are not affected by the length of the strip. The SIFs and ERRs are systematically investigated for the whole range of material combinations $\alpha \in(-1,1), \beta \in(-0.5,0.5)$. The 3D SIF distributions as well as the contour plots are shown in Figure 6 . It should be noted that all the SIFs are normalized using $\sigma \sqrt{\pi a}$ in this graph. As can be seen from Figure 6(a), the maximum value of $K_{\mathrm{I}}$ is located in the lower left and upper right corner of the $\alpha$ $\beta$ space, and the minimum $K_{\mathrm{I}}$ is located at the other two corners $(\alpha= \pm 1, \beta=0)$ of the parallelogram. In the good pair region, $K_{\mathrm{I}}$ decreases from 1.189 of the homogeneous crack 
TABLE 1: Normalized SIFs $K_{\mathrm{I}} / \sigma \sqrt{\pi a}$ and $K_{\mathrm{II}} / \sigma \sqrt{\pi a}$ for the edge interface crack $\left(\nu_{1}=\nu_{2}=0.3\right.$, plane stress).

\begin{tabular}{|c|c|c|c|c|c|}
\hline \multirow{3}{*}{$E_{1} / E_{2}$} & \multirow{3}{*}{$a / W$} & \multicolumn{4}{|c|}{ Edge interface crack } \\
\hline & & \multicolumn{2}{|c|}{ Normalized $K_{\mathrm{I}}$} & \multicolumn{2}{|c|}{ Normalized $K_{\mathrm{II}}$} \\
\hline & & Present & Matsumto et al. [21] & Present & Matsumto et al. [21] \\
\hline \multirow{4}{*}{2} & 0.1 & 1.195 & 1.190 & -0.129 & -0.127 \\
\hline & 0.2 & 1.367 & 1.367 & -0.137 & -0.137 \\
\hline & 0.3 & 1.658 & 1.657 & -0.158 & -0.156 \\
\hline & 0.4 & 2.108 & 2.109 & -0.198 & -0.195 \\
\hline \multirow{4}{*}{4} & 0.1 & 1.209 & 1.199 & -0.239 & -0.237 \\
\hline & 0.2 & 1.368 & 1.368 & -0.251 & -0.251 \\
\hline & 0.3 & 1.653 & 1.655 & -0.288 & -0.288 \\
\hline & 0.4 & 2.100 & 2.102 & -0.359 & -0.358 \\
\hline \multirow{4}{*}{10} & 0.1 & 1.229 & 1.222 & -0.340 & -0.336 \\
\hline & 0.2 & 1.369 & 1.366 & -0.349 & -0.348 \\
\hline & 0.3 & 1.648 & 1.648 & -0.399 & -0.394 \\
\hline & 0.4 & 2.089 & 2.090 & -0.495 & -0.491 \\
\hline \multirow{4}{*}{100} & 0.1 & 1.252 & 1.251 & -0.425 & -0.424 \\
\hline & 0.2 & 1.370 & 1.376 & -0.429 & -0.429 \\
\hline & 0.3 & 1.642 & 1.647 & -0.485 & -0.470 \\
\hline & 0.4 & 2.078 & 2.083 & -0.598 & -0.569 \\
\hline
\end{tabular}
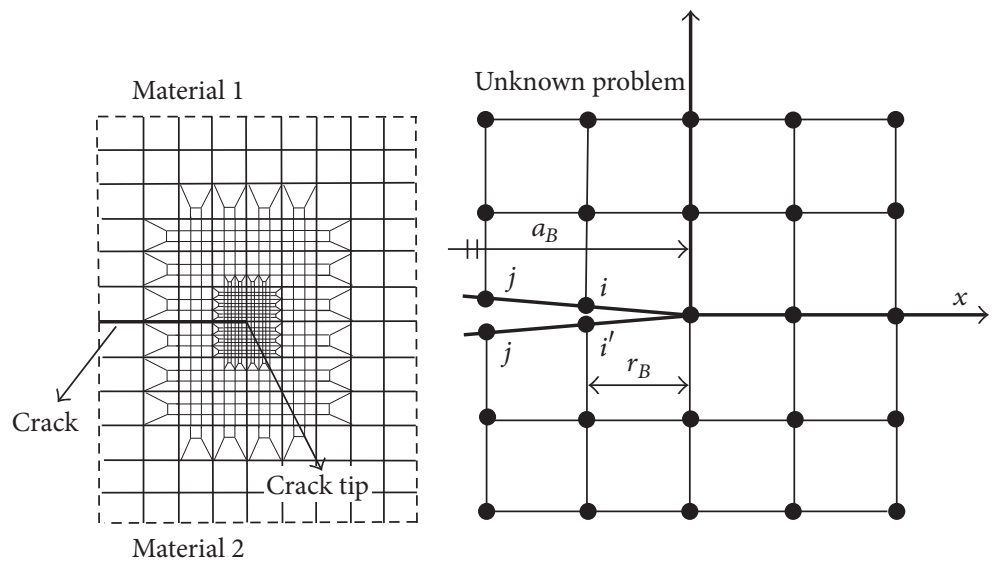

FIGURE 3: Finite element idealization around the crack tip.

( $\alpha=0, \beta=0$ ) to the diagonal lower left and upper right endpoints $(\alpha= \pm 1, \beta= \pm 0.5)$ in the $\alpha$ - $\beta$ space; conversely, it presents an increasing tendency from the origin to the other pair of diagonal corners $(\alpha= \pm 1, \beta=0)$ in the bad pair zone. In Figure $6(\mathrm{~b})$, the distributions of $K_{\mathrm{II}} / \sigma \sqrt{\pi a}$ behave almost like a plane, and the values increase from the left to the right in the contour plot monotonously. Furthermore, switching materials 1 and 2 upside down which contributes to reversed $(\alpha, \beta)$ merely changes the signs of $K_{\mathrm{II}} / \sigma \sqrt{\pi a}$, and the minimum absolute value 0 of $K_{\mathrm{II}} / \sigma \sqrt{\pi a}$ locates on the line $\beta=2.8627 \alpha$ in the whole material space.

The energy methods are widely employed in the prediction of the onset and propagation of the interface cracks or delaminations. Therefore, the strain energy release rates and their corresponding mode angles are also investigated and plotted in Figures 7(a) and 7(b), respectively. As can be seen from this graph, the normalized ERR $G E_{*} /\left(\sigma^{2} \pi a\right)$ and mode angle $\psi$ exhibit a similar varying tendency to those of the normalized SIFs $K_{\mathrm{I}} / \sigma \sqrt{\pi a}$ and $K_{\mathrm{II}} / \sigma \sqrt{\pi a}$. This is due to the fact that $K_{\mathrm{II}}$ is relatively smaller than $K_{\mathrm{I}}$ in the whole $\alpha-\beta$ space for the remote tensile loading case. It is known that the interface toughness depends strongly on the mode mixity; thus, in order to thoroughly and clearly investigate the effect of geometric configuration on the mode mixity, only the normalized ERR $G E_{*} /\left(\sigma^{2} \pi a\right)$ and mode angle $\psi$ will be demonstrated in the following sections due to the similar varying tendency between $K_{\mathrm{I}} / \sigma \sqrt{\pi a}, K_{\mathrm{II}} / \sigma \sqrt{\pi a}$, and $G E_{*} /\left(\sigma^{2} \pi a\right), \psi$. Without special notifications, one can get similar conclusions for the case of $K_{\mathrm{I}} / \sigma \sqrt{\pi a}, K_{\mathrm{II}} / \sigma \sqrt{\pi a}$.

4.3. Solutions of the Symmetrically Bonded Strip. The SIFs of the symmetrically bonded strip shown in Figure 4(a) for typical engineering materials are computed by varying the 


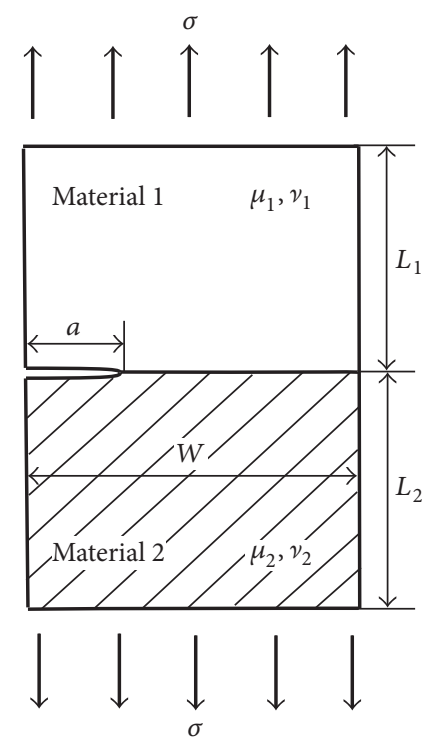

(a)

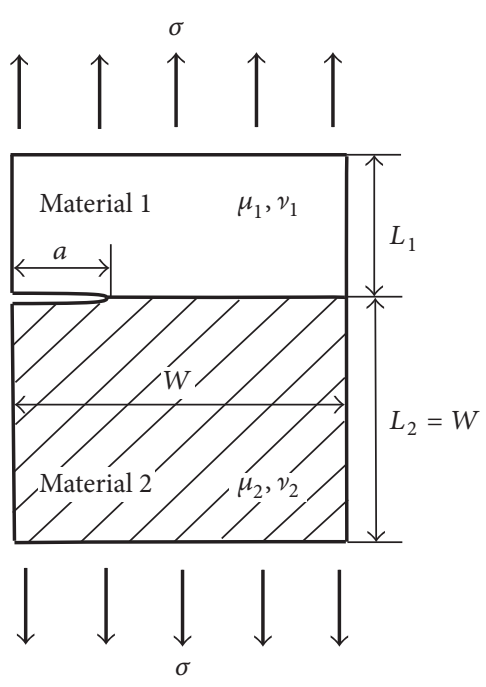

(b)

FIGURE 4: Geometric configurations for (a) longitudinal symmetrically bonded strip and (b) longitudinal asymmetrically bonded strip.

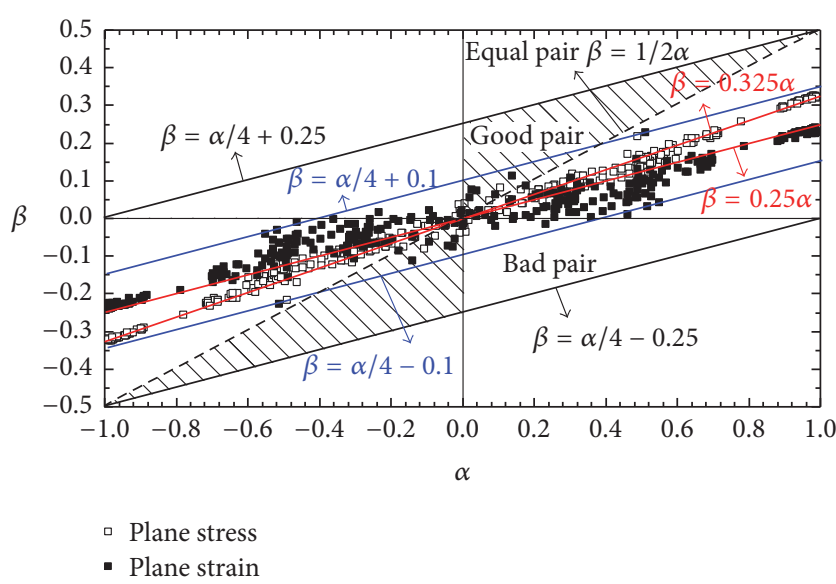

FIGURE 5: Dundurs parameters for typical engineering materials [23].

height of the two materials $L_{1}=L_{2}=0.333 \sim 2 \mathrm{~W}$. It is seen that $G E_{*} /\left(\sigma^{2} \pi a\right)$ and $\psi$ exhibit similarly varying tendency for $\beta=0.25 \alpha$ and $\beta=0.325 \alpha$ in Figures 8(a) and $8(\mathrm{~b})$, respectively. Furthermore, the effect of the height of the bonded component is divided into three different zones: $L_{1} / W \leq 0.1,0.1<L_{1} / W<0.2$, and $L_{1} / W \geq 0.2$. The dependence of ERR and mode angle on Dundurs parameter $\alpha$ exhibits different varying tendency for $L_{1} / W \leq 0.1$ and $L_{1} / W>0.2$, and the zone of $0.1<L_{1} / W \leq 0.2$ can be denoted as the transition region which connects the two different variations smoothly. In order to observe the effect of the height of the bonded component, $G E_{*} /\left(\sigma^{2} \pi a\right)$ and $\psi$ are plotted against $\alpha$ for various $L_{1} / W$ in Figures 9 (a) and 9 (b), respectively. The values of $G E_{*} /\left(\sigma^{2} \pi a\right)$ for $L_{1} / W \leq 0.1$ and $\beta=0.325 \alpha$ are plotted in red in the right ordinates in Figure 9 (a) to make the variations obvious. Figure 9 (a) shows that the maximum and minimum values of $G E_{*} /\left(\sigma^{2} \pi a\right)$ for $L_{1} / W \leq 0.1$ are located at $\alpha=0$ and $\alpha \rightarrow \pm 1$, respectively. However, opposite trends are observed when $L_{1} / W>0.2$. Furthermore, Figure 9(b) shows that the maximum and minimum $\operatorname{abs}(\psi)$ are always located at $\alpha \rightarrow \pm 1$ and $\alpha=0$, respectively, for a given height of $L_{1} / W>0.2$. This means that the severe mismatch in the plane tensile modulus across the interface would lead to a bigger mode angle in this case, and $\psi$ does not change monotonically with $\alpha$ for the other two zones of $L_{1} / W \leq 0.1$ and $0.1<L_{1} / W<0.2$.

4.4. Solutions of the Asymmetrically Bonded Strip. The normalized ERR $G E_{*} /\left(\sigma^{2} \pi a\right)$ and its corresponding mode angle $\psi$ of the edge-cracked asymmetrically bonded strip shown in Figure 4(b) under the material combinations of $\beta=0.25 \alpha$ and $\beta=0.325 \alpha$ are computed by varying the height of the two materials $L_{1}=0.333 \sim 2 W$. As demonstrated in Figure 10, a similar varying tendency for $\beta=0.25 \alpha$ and $\beta=0.325 \alpha$ also exists in the asymmetrically bonded strip. Similarly, $G E_{*} /\left(\sigma^{2} \pi a\right)$ and $\psi$ for some typical $L_{1} / W$ are plotted against $\alpha$ in Figures 11(a) and 11(b), respectively. It should be noted that $G E_{*} /\left(\sigma^{2} \pi a\right)$ of $L_{1} / W \leq 0.1$ and $\beta=0.325 \alpha$ are plotted in red in the right ordinates of Figure 11(a) to make the variations obvious. Three different zones, that is, $L_{1} / W \leq 0.1,0.1<$ $L_{1} / W<0.2$, and $L_{1} / W \geq 0.2$, are also employed in the discussion. Firstly, considering the case where $L_{1} / W \leq 0.1$, when material 1 in Figure 4(b) is extremely stiff compared to material 2, $\alpha$ approaches $1, G E_{*} /\left(\sigma^{2} \pi a\right)$ in Figure 11(a) reaches its minimum value, and $\operatorname{abs}(\psi)$ in Figure 11(b) comes closer to its maximum one. However, when material 1 is extremely compliant compared to material 2, $\alpha$ approaches -1 and $G E_{*} /\left(\sigma^{2} \pi a\right)$ and $\psi$ reach their maximum and minimum values, respectively. Furthermore, the maximum $\operatorname{abs}(\psi)$ is always located around $\alpha \rightarrow-1$. Secondly, considering 

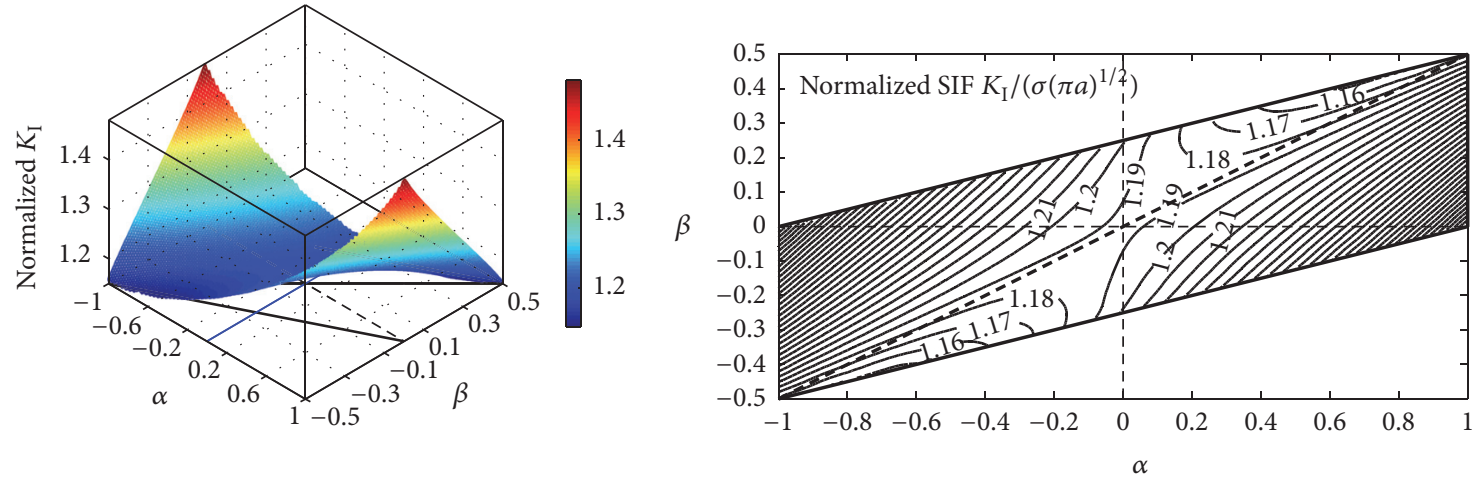

(a)
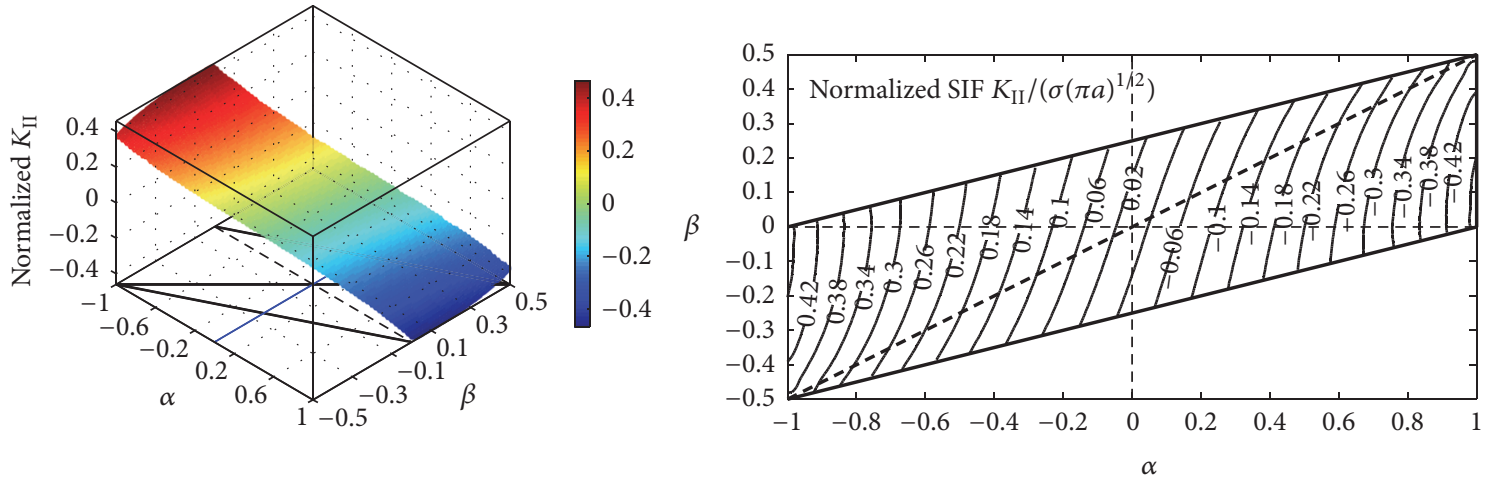

(b)

FIGURE 6: 3D normalized SIFs and contour plots of (a) $K_{\mathrm{I}} / \sigma \sqrt{\pi a}$ and (b) $K_{\mathrm{II}} / \sigma \sqrt{\pi a}$.
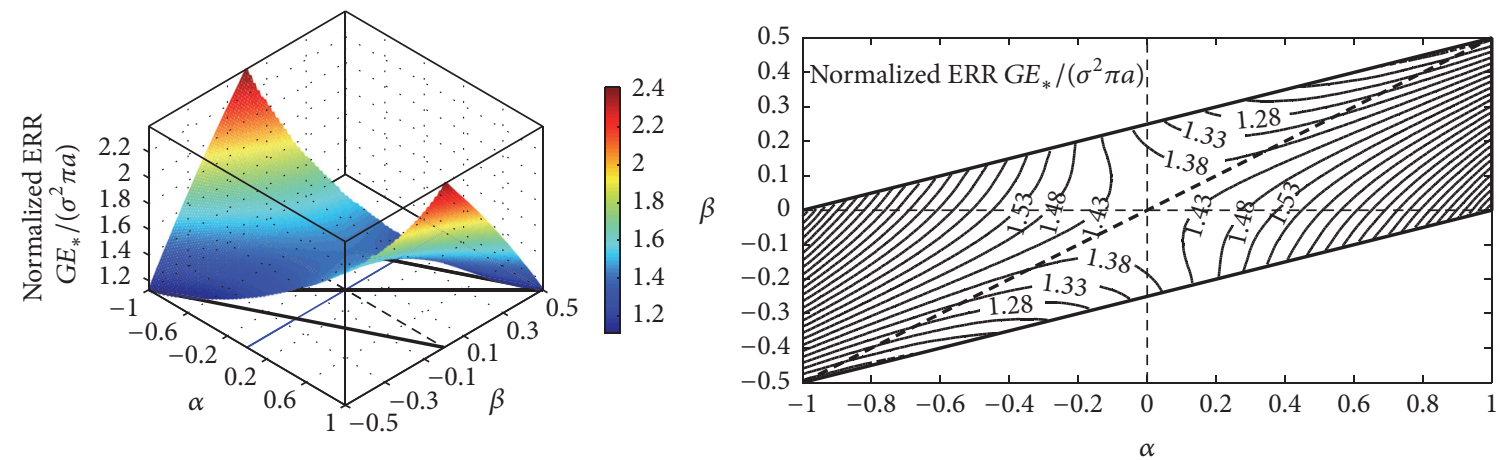

(a)
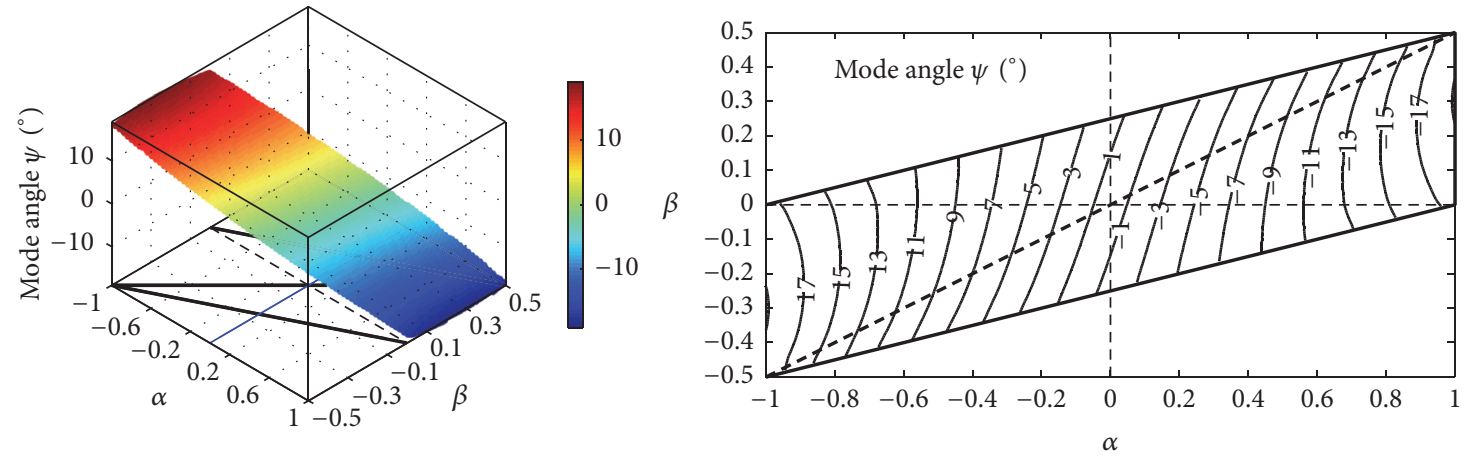

(b)

FIGURE 7: 3D distributions and contour plots of (a) normalized ERR $G E_{*} /\left(\sigma^{2} \pi a\right)$ and (b) mode angle $\psi$. 

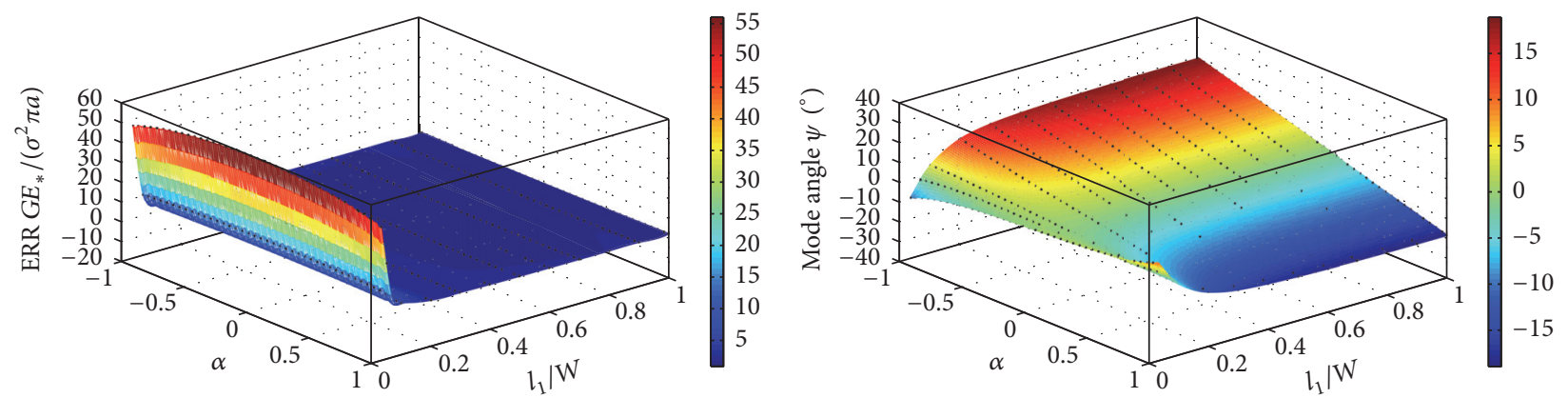

(a)
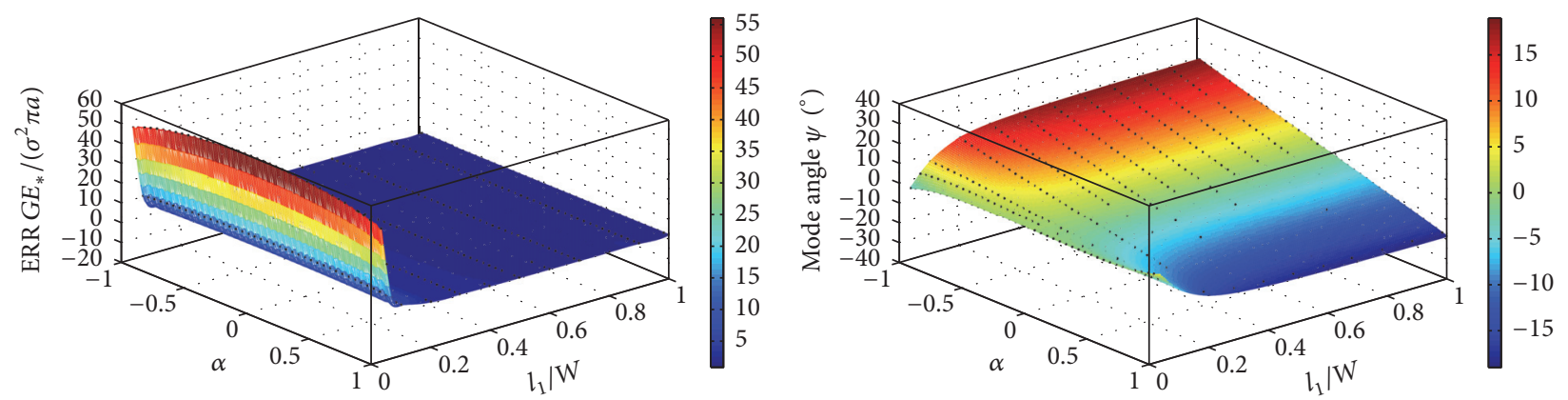

(b)

FIGURE 8: Normalized ERR $G E_{*} /\left(\sigma^{2} \pi a\right)$ and mode angle $\psi$ of a symmetrically bonded strip for (a) $\beta=0.25 \alpha$ and (b) $\beta=0.325 \alpha$.

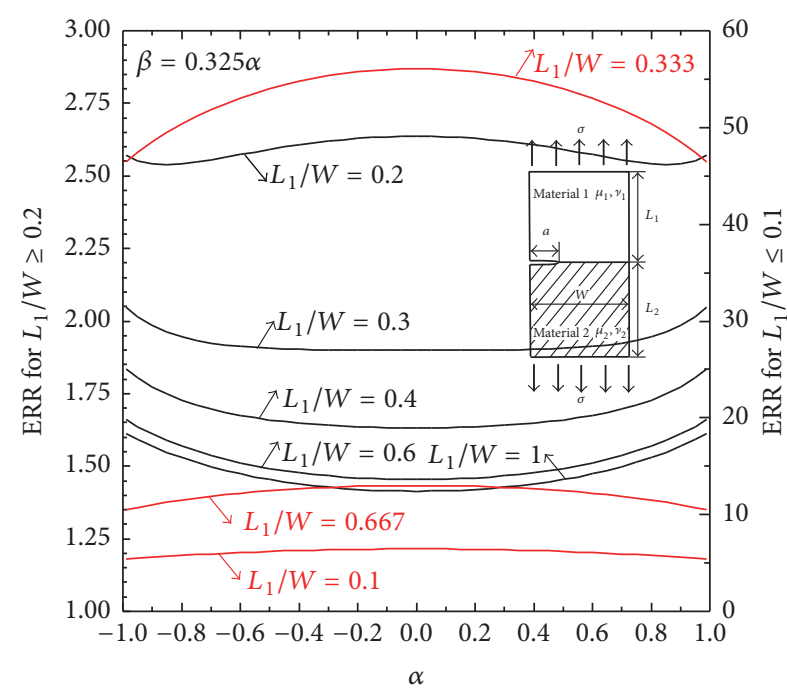

(a)

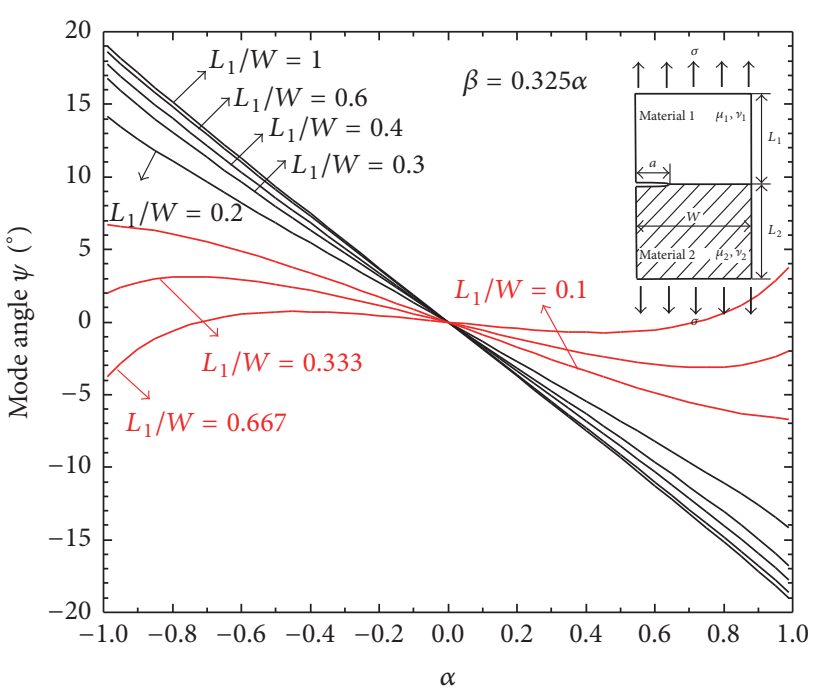

(b)

FIGURE 9: Variations of (a) normalized ERR $G E_{*} /\left(\sigma^{2} \pi a\right)$ and (b) mode angle $\psi$ of a symmetrically bonded strip for $\beta=0.325 \alpha$.

the case where $L_{1} / W \geq 0.2$, Figure 11(a) shows that the minimum $G E_{*} /\left(\sigma^{2} \pi a\right)$ is located at $\alpha \in(0,1)$, and increasing $L_{1} / W$ moves the minimum $G E_{*} /\left(\sigma^{2} \pi a\right)$ towards $\alpha=0$. Furthermore, when material 1 (lower height) is extremely compliant compared to material $2(\alpha \rightarrow-1), G E_{*} /\left(\sigma^{2} \pi a\right)$ reaches its maximum value for a specific $L_{1} / W$. Finally, for the case of $0.1<L_{1} / W<0.2$, the normalized energy release rate $G E_{*} /\left(\sigma^{2} \pi a\right)$ is determined by the combined effect of the aforementioned discussions. The exact size of the transition zone is dominated by the constraint of the remote load on the singular stress field around the crack tip.

4.5. Effect of the Height of the Bonded Components on ERR and Mode Mixity. The variations of the normalized ERR $G E_{*} /\left(\sigma^{2} \pi a\right)$ and mode angle $\psi$ are plotted against logarithmic $L_{1} / W$ with varying $\alpha=-0.9,0.45,0,0.45$, and 0.9 in Figures 12(a) and 12(b), respectively. The lines of the symmetrical models are plotted in black and those 

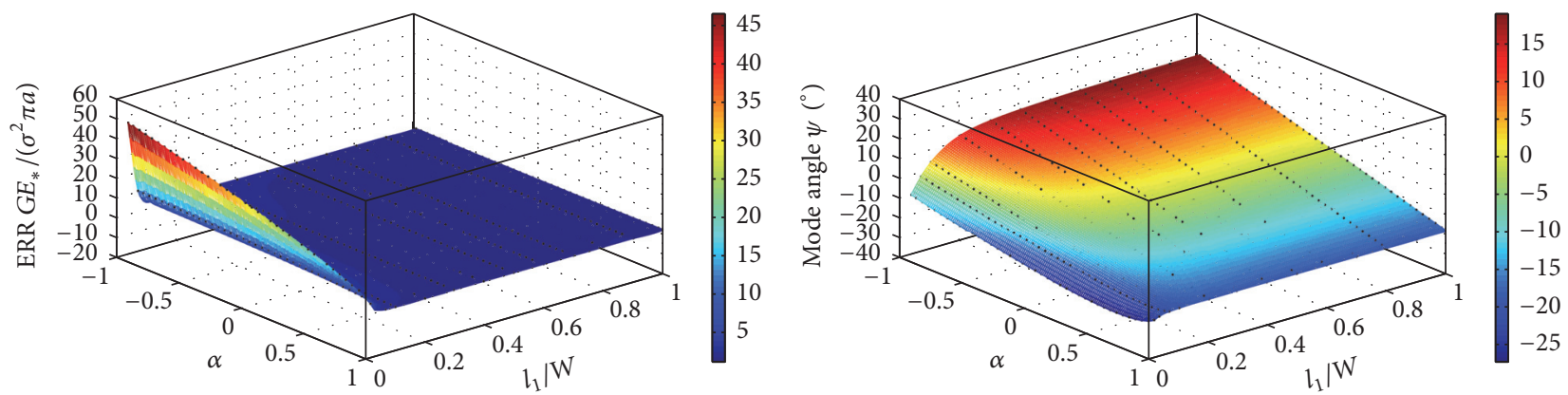

(a)
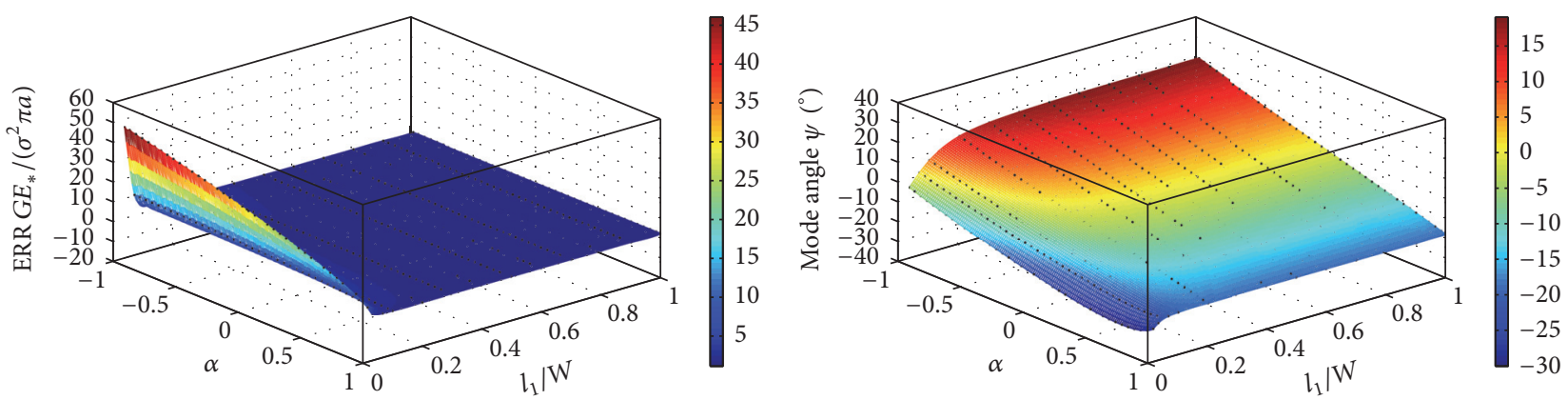

(b)

Figure 10: Normalized ERR $G E_{*} /\left(\sigma^{2} \pi a\right)$ and mode angle $\psi$ of an asymmetrically bonded strip for (a) $\beta=0.25 \alpha$ and (b) $\beta=0.325 \alpha$.

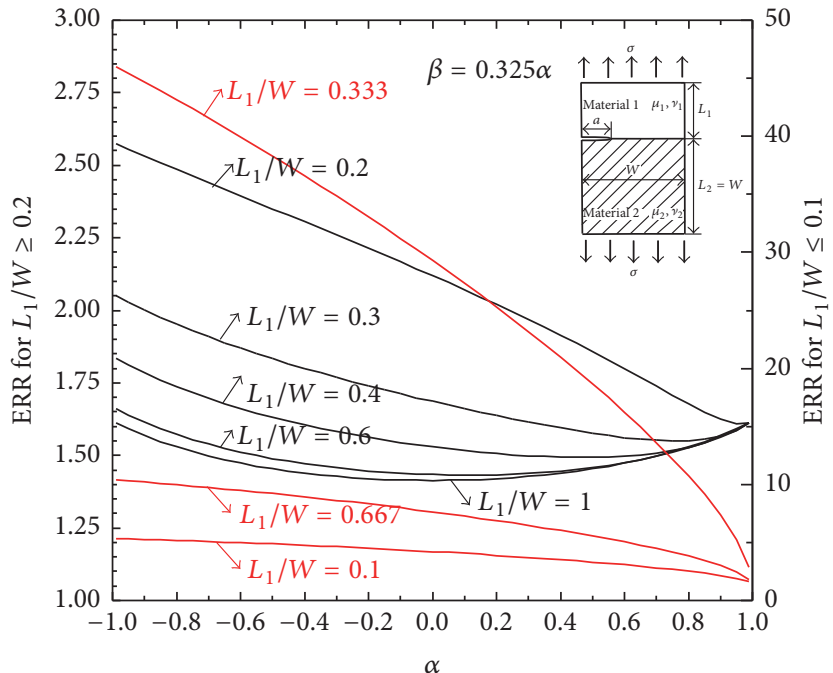

(a)

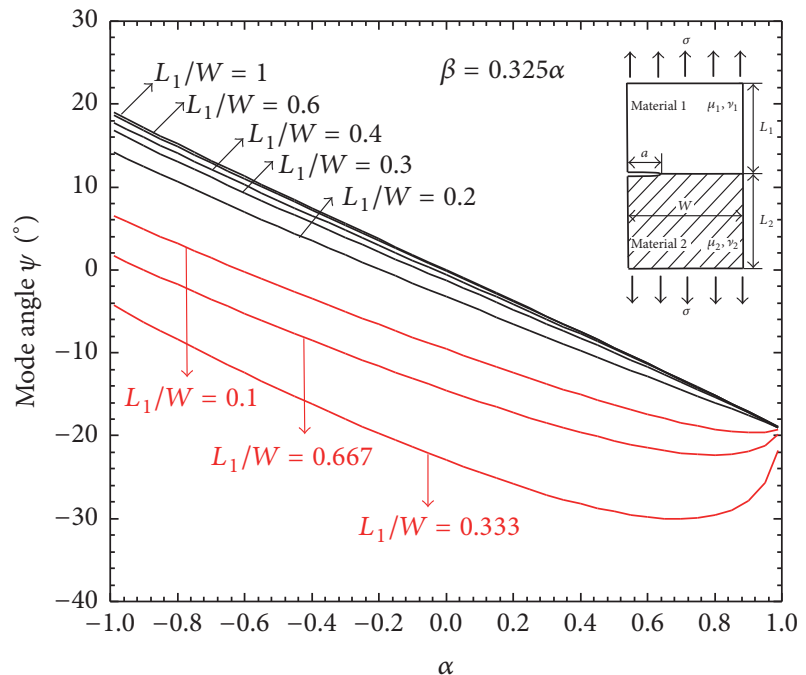

(b)

FIGURE 11: Variations of (a) normalized ERR $G E_{*} /\left(\sigma^{2} \pi a\right)$ and (b) mode angle $\psi$ of an asymmetrically bonded strip under $\beta=0.325 \alpha$.

of the asymmetrical models are in red. The effects of the height of the bonded components on ERR and mode mixity for each model are investigated. Then, a schematic of the simple elliptic rule fracture criterion shown in Figure 13 is employed to predict the possibility of crack generation for a demonstration. Furthermore, the elastic behaviors of the symmetrical and asymmetrical models are compared and discussed.

Firstly, let us consider the symmetrically bonded strip shown in Figure 4(a). Figure 12(a) shows that $G E_{*} /\left(\sigma^{2} \pi a\right)$ decreases monotonously and sharply with increasing height $L_{1}$ for fixed $\alpha$ when $L_{1} / W \leq 0.1$, and it then gradually increases and reaches a constant when $L_{1} \geq W$. Furthermore, as demonstrated in Figure 12(b), the mode angles $\psi$ are opposite in sign and equal in magnitude for the symmetric model, and the absolute values abs $(\psi)$ decrease with decreasing $L_{1}$ from a constant value at $L_{1} \geq W$.

Secondly, considering the other case of the asymmetrical model shown in Figure 4(b), $G E_{*} /\left(\sigma^{2} \pi a\right)$ increases with decreasing $L_{1}$ for fixed $\alpha$, and $\psi$ exhibits a simple decreasing 


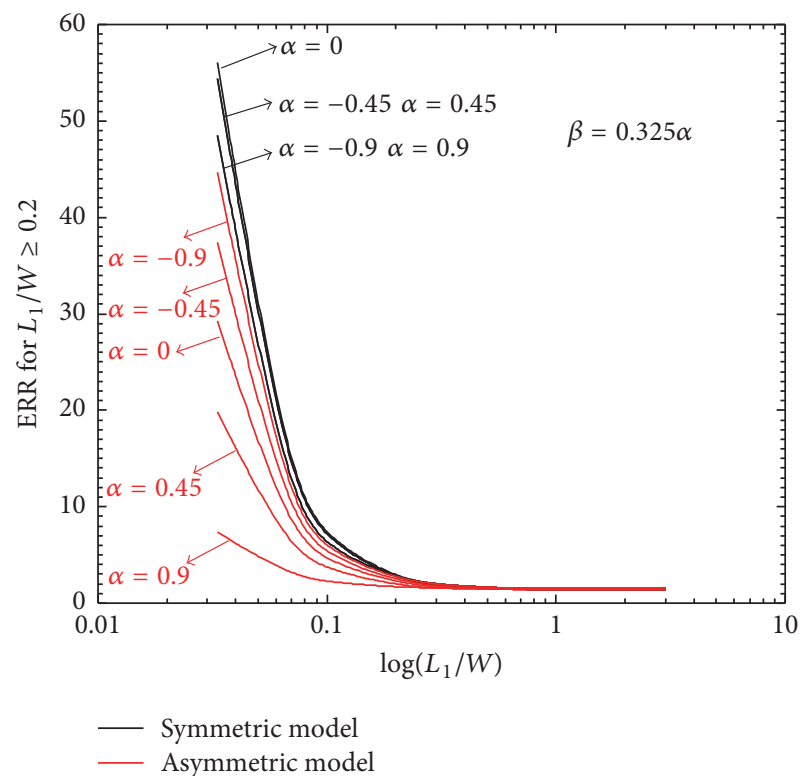

(a)

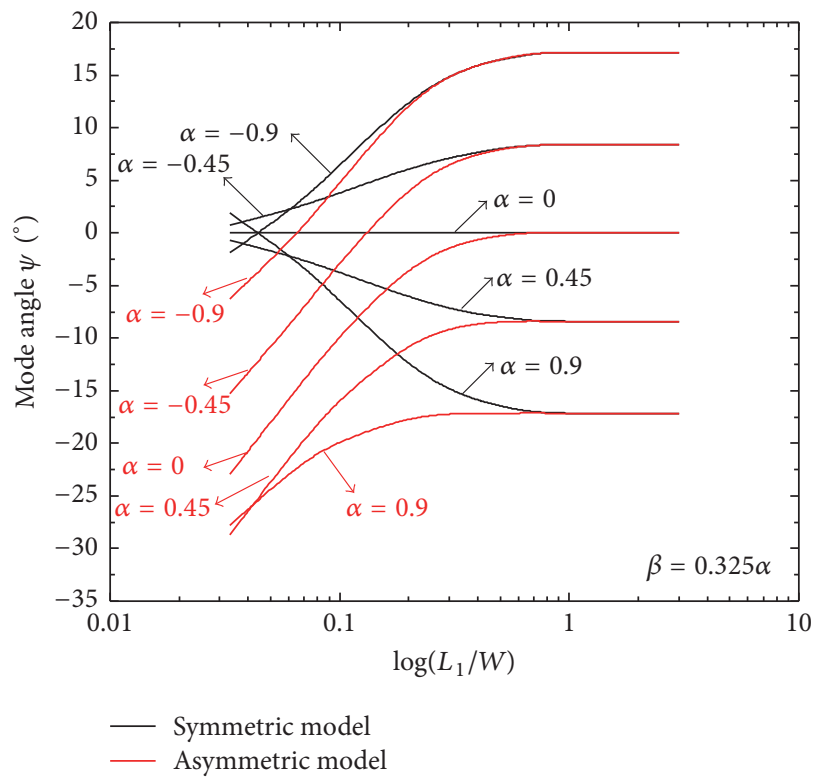

(b)

FIGURE 12: Variations of (a) normalized ERR $G E_{*} /\left(\sigma^{2} \pi a\right)$ and (b) mode angle $\psi$ against logarithmic $L_{1} / W$.

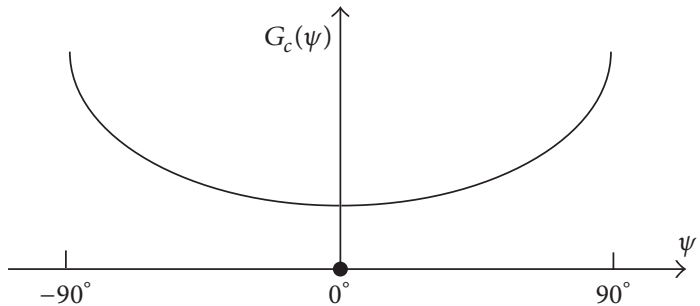

FIGURE 13: Variation of $G_{C}(\psi)$ with $\psi$ for the typically elliptic rule fracture criterion.

tendency with decreasing $L_{1}$; Figure 12(b) also demonstrates that the absolute value abs $(\psi)$ does not exhibit a uniform varying relationship for all the material combinations. Specifically, when $\alpha \geq 0$, the mode angle $\psi \leq 0$ and its magnitude increases with decreasing $L_{1}$; however, when $\alpha<0$, in the process of $L_{1}$ getting smaller, the sign of $\psi$ changes from positive to negative and the magnitude of $\psi$ increases after an initial decrease.

Finally, the elastic behaviors of the symmetrical and asymmetrical models are compared. $G E_{*} /\left(\sigma^{2} \pi a\right)$ of the symmetrical model is always bigger than that of the asymmetrical model for fixing $L_{1}, \alpha$. However, the interface toughness (say critical ERR or critical SIFs) depends strongly on the mode mixity $\psi$. The variation of the critical ERR $G_{C}(\psi)$ with $\psi$ for the typically elliptic rule fracture criterion is demonstrated in Figure 13. It should be noted that the asymmetry in the interface toughness as well as the minimum toughness deviated from the origin $(\psi \neq 0)$ may exist for specific material combinations. Here, we assume that small magnitude in $\psi$ means smaller interface toughness for this specific rough criterion. Therefore, the elastic behaviors for different geometric configurations should be compared together with the mode mixity. When material 1 becomes stiffer $\alpha \geq 0, G E_{*} /\left(\sigma^{2} \pi a\right)$ of the symmetrical model gets higher, and its mode angle $\psi$ becomes smaller which means smaller interface toughness. Therefore, the symmetrical model faces a higher risk of crack initiation compared with the asymmetrical model. On the other hand, when material 1 is more compliant $\alpha<0$, the magnitude of $\psi$ of the symmetrical model increases after an initial decrease with decreasing $L_{1}$. Therefore, in the first stage of decreasing $\psi$, the symmetrical model is also more dangerous than the asymmetrical one. However, in the next stage of increasing $\psi$, the elastic behavior is dominated by the combined effect of material combination and geometrical configuration. The relationship between the two models should be verified through a detailed comparison of the specific values between the ERR and the critical interface toughness.

\section{Conclusions}

In this research, the elastic behavior of an edge-cracked dissimilar bonded strip subjected to remote tensile load is investigated with varying different geometric configurations for arbitrary material combinations. The stress intensity factors, energy release rates, and mode angles are computed and compared systematically. It was found that the dependence of SIF, ERR, and mode angle on material combination should be discussed in three different zones according to the height of the bonded component. Furthermore, the combined effects of material combination and the geometric configurations on the elastic behavior of the bimaterial bonded strip are also investigated. Then, the combined effects of the relative height of each bonded component and material combination are 
discussed for the typical engineering materials. This may help the guidance for material selection and structural design for the modern layered materials.

\section{Conflicts of Interest}

The authors declare that there are no conflicts of interest regarding the publication of this paper.

\section{Acknowledgments}

This work was supported in part by grants from the National Natural Science Foundation of China (NSFC) (no. 51376111) and the Fundamental Research Funds of Shandong University (no. 2016TB009).

\section{References}

[1] M. L. Williams, "The stresses around a fault or crack in dissimilar media," Bulletin of the Seismological Society of America, vol. 49, no. 2, pp. 199-204, 1959.

[2] J. R. Rice and G. C. Sih, "Plane problems of cracks in dissimilar media," Journal of Applied Mechanics, vol. 32, no. 2, pp. 418-423, 1965.

[3] F. Erdogan, "Stress distribution in a nonhomogeneous elastic plane with cracks," Journal of Applied Mechanics, vol. 30, no. 2, pp. 232-236, 1963.

[4] F. Erdogan, "Stress distribution in bonded dissimilar materials with cracks," Journal of Applied Mechanics, vol. 32, no. 2, pp. 403-409, 1965.

[5] A. H. England, "A crack between dissimilar media," Journal of Applied Mechanics, vol. 32, no. 2, pp. 400-402, 1965.

[6] J. R. Willis, "Crack propagation in viscoelastic media," Journal of the Mechanics \& Physics of Solids, vol. 15, no. 4, pp. 229-240, 1967.

[7] M. L. Williams, "Stress singularities resulting from various boundary conditions in angular corners of plates in extension," Journal of Applied Mechanics, vol. 19, pp. 526-528, 1952.

[8] D. B. Bogy, "Two edge-bonded elastic wedges of different materials and wedge angles under surface tractions," Journal of Applied Mechanics, vol. 38, no. 2, pp. 377-386, 1971.

[9] D. B. Bogy and K. C. Wang, "Stress singularities at interface corners in bonded dissimilar isotropic elastic materials," International Journal of Solids and Structures, vol. 7, no. 8, pp. 9931005, 1971.

[10] V. L. Hein and F. Erdogan, "Stress singularities in a two-material wedge," International Journal of Fracture Mechanics, vol. 7, no. 3, pp. 317-330, 1971.

[11] J. P. Dempsey and G. B. Sinclair, "On the stress singularities in the plane elasticity of the composite wedge," Journal of Elasticity, vol. 9, no. 4, pp. 373-391, 1979.

[12] J. C. W. van Vroonhoven, "Stress singularities in bi-material wedges with adhesion and delamination," Fatigue \& Fracture of Engineering Materials \& Structures, vol. 15, no. 2, pp. 159-171, 1992.

[13] E. D. Reedy Jr. and T. R. Guess, "Composite-to-metal tubular lap joints: strength and fatigue resistance," International Journal of Fracture, vol. 63, no. 4, pp. 351-367, 1993.

[14] J.-Q. Xu, Y.-H. Liu, and X.-G. Wang, "Numerical methods for the determination of multiple stress singularities and related stress intensity coefficients," Engineering Fracture Mechanics, vol. 63, no. 6, pp. 775-790, 1999.

[15] A. R. Akisanya and N. A. Fleck, "Interfacial cracking from the freeedge of a long bi-material strip," International Journal of Solids and Structures, vol. 34, no. 13, pp. 1645-1665, 1997.

[16] X. Lan, N.-A. Noda, K. Mithinaka, and Y. Zhang, "The effect of material combinations and relative crack size to the stress intensity factors at the crack tip of a bi-material bonded strip," Engineering Fracture Mechanics, vol. 78, no. 14, pp. 2572-2584, 2011.

[17] N.-A. Noda and X. Lan, "Stress intensity factors for an edge interface crack in a bonded semi-infinite plate for arbitrary material combination," International Journal of Solids and Structures, vol. 49, no. 10, pp. 1241-1251, 2012.

[18] X. Lan, S. B. Ji, N. A. Noda, and Y. Cheng, "Stress intensity factor solutions for several crack problems using the proportional crack opening displacements," Engineering Fracture Mechanics, vol. 171, no. 15, pp. 35-49, 2017.

[19] B. M. Malyshev and R. L. Salganik, "The strength of adhesive joints using the theory of cracks," International Journal of Fracture Mechanics, vol. 1, no. 2, pp. 114-128, 1965.

[20] J. R. Rice, "Elastic fracture mechanics concepts for interfacial cracks," Journal of Applied Mechanics, Transactions ASME, vol. 55, no. 1, pp. 98-103, 1988.

[21] T. Matsumto, M. Tanaka, and R. Obara, "Computation of stress intensity factors of interface cracks based on interaction energy release rates and BEM sensitivity analysis," Engineering Fracture Mechanics, vol. 65, no. 6, pp. 683-702, 2000.

[22] MSC Marc 2007, MSC Marc 2007 User's Guide, MSC Software Corporation, Santa Ana, Calif, USA, 2007.

[23] T. Suga, G. Elssner, and S. Schmauder, "Composite parameters and mechanical compatibility of material joints," Journal of Composite Materials, vol. 22, no. 10, pp. 917-934, 1988.

[24] J. Dundurs and A. C. Gangadharan, "Edge dislocation near an inclusion with a slipping interface," Journal of the Mechanics and Physics of Solids, vol. 17, no. 6, pp. 459-471, 1969. 

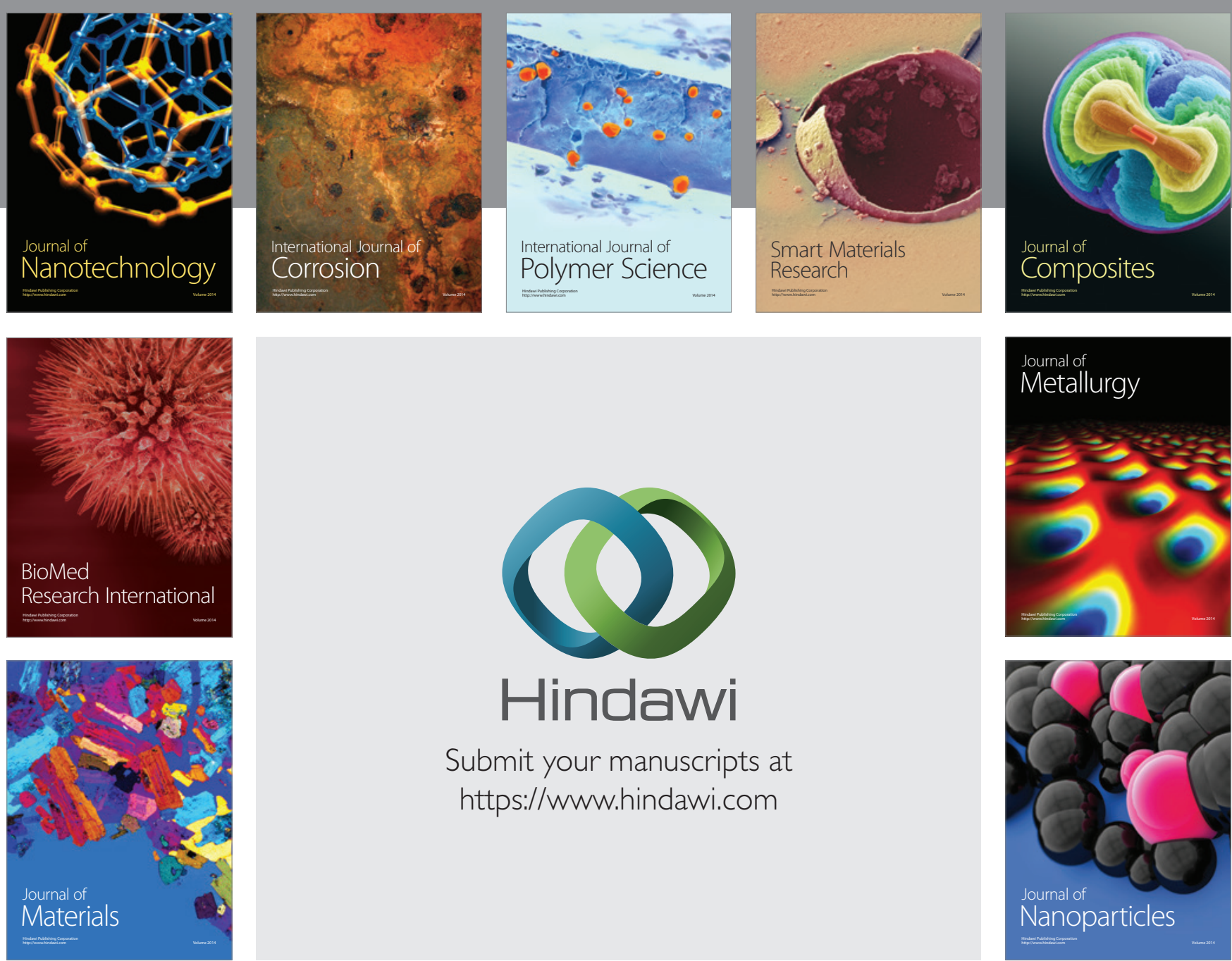

\section{Hindawi}

Submit your manuscripts at

https://www.hindawi.com

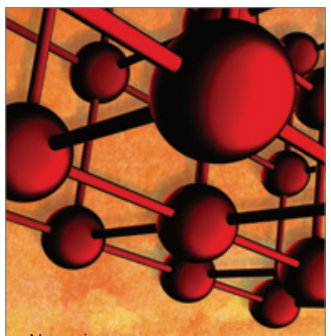

Materials Science and Engineering
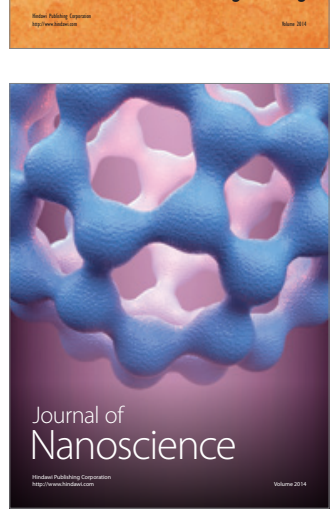
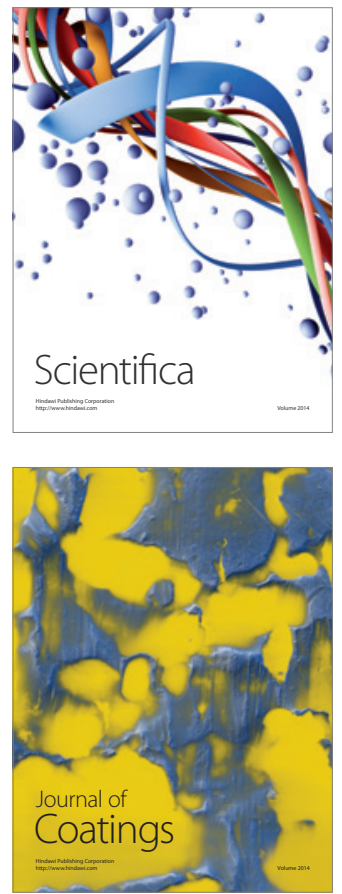
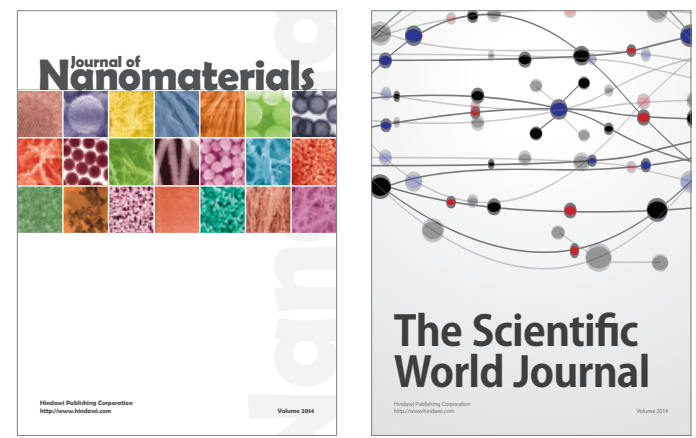

The Scientific World Journal
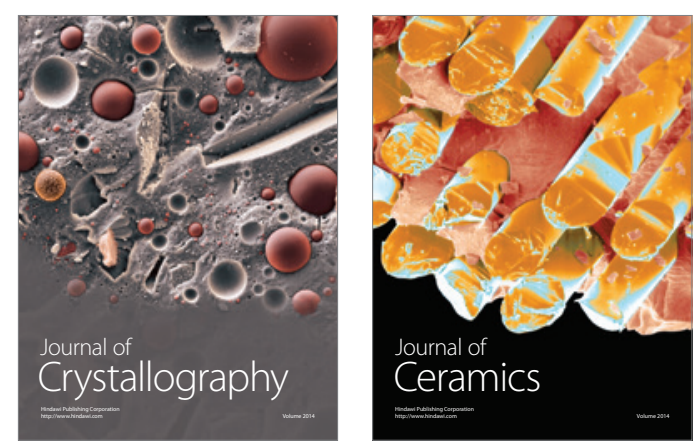
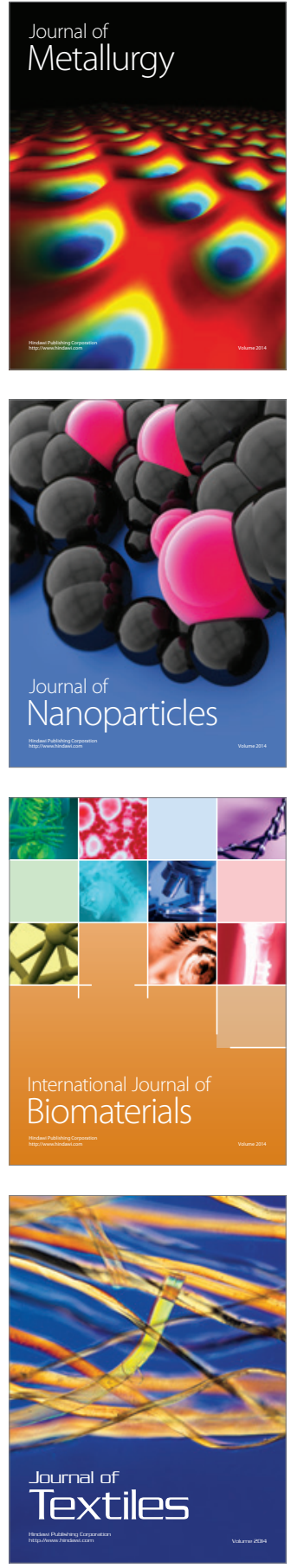